\title{
Epiteliopatías corneales subdiagnosticadas: epiteliopatías tóxica, nutricional y diabética
}

\section{Corneal epitheliopathy under diagnosed: Toxic, nutricional and diabetic epitheliopathy}

\author{
Arturo Ramírez-Miranda* y Ricardo Blas-Medina
}

Departamento de Córnea y Cirugía Refractiva, Instituto de Oftalmología «Fundación Conde de Valenciana» IAP, Ciudad de México, México

\section{Resumen}

Existen muchas causas de epiteliopatías corneales; sin embargo, las causas poco frecuentes son infradiagnosticadas debido al poco conocimiento que existe acerca de ellas. Dentro de éstas la más frecuente es, sin duda, la epiteliopatía tóxica, debida al mal uso o sobretratamiento de alguna medicación tópica. Ejerce sus efectos tóxicos directamente ocasionando una queratopatía punteada superficial o indirectamente provocando inflamación e interfiriendo en la migración de células basales epiteliales del limbo. Existe un amplio rango de agentes que pueden provocar epiteliopatía tóxica, el más conocido de los cuales es el cloruro de benzalconio (BAK), altamente tóxico en el epitelio corneal y fuertemente asociado con epiteliopatía tóxica, conjuntivitis alérgica y blefaritis. En la literatura se han reportado epiteliopatías tóxicas secundarias a quimioterapias específicamente con anticuerpos monoclonales que inhiben el receptor del factor de crecimiento epidérmico (EGFR). En cuanto a las causas nutricionales, la epiteliopatía secundaria a deficiencia de vitamina $A$ es un aspecto clínico muy importante en esta enfermedad, que ocasiona una inestabilidad de la capa lagrimal precorneal provocando una epiteliopatía punteada, que puede progresar a defectos epiteliales, edema estromal y queratinización. La diabetes mellitus (DM) puede llevar a varias complicaciones oculares, entre las cuales se encuentra la epiteliopatía diabética que comprende la queratopatía punteada superficial y defectos epiteliales persistentes, con un impacto clínico importante debido a los síntomas que produce, desde sensación de cuerpo extraño hasta baja visual. Es importante conocer este tipo de enfermedades para su adecuado diagnóstico y tratamiento. Deben buscarse intencionadamente en la práctica oftalmológica en los casos de epiteliopatías persistentes o refractarias al tratamiento convencional.

Palabras clave: Epitelio corneal. Compuestos de benzalconio. Conjuntivitis alérgica. Blefaritis. Inflamación. Diabetes mellitus.

\begin{abstract}
There are many causes of corneal epitheliopathies, however, the rare causes are underdiagnosed due to the little knowledge that exists about them. Among these the most frequent is undoubtedly the toxic epitheliopathy, due to the misuse or treatment of some topical medication, exerting its toxic effects directly to cause a superficial punctate keratopathy, or indirectly causing inflammation and interfering with the migration of the epithelial limbal cells. There is a wide range of agents that can cause toxic epitheliopathy, the most known benzalkonium chloride (BAK), highly toxic to the corneal epithelium, strongly associated with toxic epitheliopathy, allergic conjunctivitis and blepharitis. There have been described toxic epithelopathies secondary to chemotherapy specifically with monoclonal antibodies that inhibit the epidermal growth factor receptor (EGFR). The epitheliopathy secondary to vitamin A deficiency is a very important clinical aspect in this pathology, causing an instability of

Correspondencia:

*Arturo Ramírez-Miranda

Chimalpopoca, 14

Fecha de recepción: 04-10-2017

Fecha de aceptación: 20-02-2018 DOI: 10.24875/RMO.M18000036

Disponible en internet: 02-07-2018 Rev Mex Oftalmol. 2018;92(4):173-179 www.rmo.com.mx cia CC BY-NC-ND (http://creativecommons.org/licenses/by-nc-nd/4.0/).
\end{abstract}


the precorneal tear layer causing a punctate epitheliopathy, which can progress to epithelial defects, stromal edema and keratinization. Diabetes mellitus can lead to several ocular complications, among which is the diabetic epitheliopathy that includes superficial punctate keratopathy and persistent epithelial defects, having an important clinical impact due to the symptoms produced from foreign body sensation to visual impairment. It is important to know this entities for its proper diagnosis and treatment. Search intentionally in ophthalmological practice in cases of persistent epitheliopathies or treatment resistant.

Key words: Corneal epithelium. Benzalkonium compounds. Allergic conjunctivitis. Blepharitis. Inflammation. Diabetes mellitus.

\section{Introducción}

La epiteliopatía corneal tiene una gran variedad de causas y de presentaciones clínicas, lo cual genera un reto en la práctica oftalmológica. Las causas poco frecuentes están infradiagnosticadas debido al poco conocimiento que se tiene acerca de ellas. Por ello, es de suma importancia conocerlas y diagnosticarlas oportunamente para un tratamiento eficaz.

\section{Epiteliopatía tóxica}

La medicación tópica es un tratamiento muy específico en la práctica clínica oftalmológica. Las gotas oftálmicas tienen numerosos factores que pueden afectar a la superficie ocular. Debido a que el volumen de una gota es demasiado para el saco conjuntival, los componentes de la lágrima, incluyendo electrólitos, proteínas y mucina, son removidos de la película lagrimal. Estas gotas pueden alterar también el pH y la presión osmótica. Además, se sabe que inhiben la proliferación, regeneración y renovación del epitelio corneal ${ }^{1}$.

La automedicación es una práctica común en México, especialmente en personas de bajo nivel socioeconómico que raramente buscan un especialista para un manejo apropiado de sus problemas médicos. Aunado a esto, algunos pacientes toman prestados medicamentos tópicos de familiares o amigos. Cuando los aplican y obtienen resultados no esperados, los pacientes buscan ayuda profesional.

Se ha demostrado que el riesgo de presentar reacciones adversas de los medicamentos está relacionado con el número de medicamentos prescritos ${ }^{2}$. Consecuentemente, el uso de múltiples medicamentos aumenta la probabilidad de interacciones medicamentosas, toxicidad y combinaciones que pueden ser sinérgicas. Sin embargo, los mismos oftalmólogos pueden ser los culpables del sobretratamiento de los pacientes, con ciertos medicamentos tópicos que pueden tener efectos deletéreos en los tejidos oculares.

\section{Patofisiología}

Una sustancia tóxica puede definirse como cualquier sustancia que por su reacción química provoca daño estructural o alteración en la función, por encima de cualquier efecto terapéutico ${ }^{3}$. Los medicamentos pueden ser directamente tóxicos en el epitelio corneal, dañando su estructura y alterando su función, acompañándose 0 no de una respuesta inflamatoria ${ }^{4}$. La mayoría de estos medicamentos ejercen sus efectos tóxicos mediante múltiples mecanismos propuestos: directamente al ocasionar una queratopatía punteada superficial o indirectamente provocando inflamación mediante infiltrados estromales en forma de anillo como en los casos de sobreuso de anestésicos o antiinflamatorios ${ }^{5}$. Algunos medicamentos tópicos pueden interferir con la migración de células basales epiteliales del limbo, especialmente si existen defectos epiteliales, ocasionando un retraso en la epitelización y promoviendo infiltrados estromales.

Las gotas oftálmicas están compuestas por elfármaco activo, así como por conservadores y soluciones buffer. El BAK se ha usado como conservador por muchos años ${ }^{6}$. Es muy soluble en agua y fácil de manejar. Debido a que el BAK tiene una fuerte actividad antibacterial, estas gotas pueden estar seguras por periodos de tiempo muy largos. Sin embargo, el BAK es altamente tóxico en el epitelio corneal y está fuertemente asociado con epiteliopatía tóxica y conjuntivitis alérgica o blefaritis?

\section{Toxicidad por medicamentos oftálmicos}

La toxicidad en el epitelio corneal secundaria a medicamentos tópicos comúnmente es posterior al uso de medicamentos antiglaucomatosos, anestésicos y, en menor grado, antibióticos tópicos ${ }^{5,8}$ (Fig. 1).

Los antiinflamatorios no esteroideos (AINE) también pueden causar complicaciones severas en la superficie ocular $^{9}$. Existe un amplio rango de agentes que pueden provocar epiteliopatía tóxica; sin embargo, hay algunos más conocidos por esta capacidad, incluyendo 




Figura 1. Imagen de la superficie corneal (A) y con tinción de fluoresceína (B) de un paciente con piteliopatía tóxica secundaria a gotas tópicas de ciprofloxacino al $0.3 \%$ (tomada del archivo de fotos del Servicio de Córnea y Cirugía Refractiva del Instituto de Oftalmología "Fundación Conde de Valenciana»).

antibióticos como aminoglucósidos (neomicina y gentamicina), fluoroquinolonas (especialmente ciprofloxacino), hipotensores oculares (especialmente timolol y dorzolamida), AINE (particularmente diclofenaco) y anestésicos tópicos. Los conservadores oftálmicos como el timerosal y el BAK se han asociado ampliamente a la epiteliopatía tóxica. Mientras que el timerosal se ha retirado de la mayoría de los medicamentos oftálmicos y soluciones de lentes de contacto en EE.UU., el BAK es el conservador más comúnmente empleado en los fármacos oftálmicos, a pesar de su reconocido potencial de hipersensibilidad ${ }^{4}$.

\section{Toxicidad por otras causas}

Puede existir epiteliopatía tóxica como resultado del contacto de manera accidental o no deseada de sustancias cáusticas con la superficie ocular. En esta variedad de sustancias se hallan desde agentes que se pueden encontrar en el hogar (cloro, amonio, alcohol y queroseno) hasta armas químicas (gas pimienta [Oleoresin capsicum] o gas lacrimógeno [ortoclorobenzilideno malononitrilo]) y sustancias tóxicas producidas por animales o plantas (veneno, toxinas de plantas y animales) $)^{2,10}$. Es menos probable que estos agentes provoquen una reacción de hipersensibilidad tipo I y más comúnmente provoquen un daño en las células epiteliales por toxicidad directa, seguida de efectos inflamatorios secundarios ${ }^{11}$.

En la literatura se han reportado epiteliopatías y perforaciones corneales secundarias a quimioterapias específicamente con anticuerpos monoclonales que inhiben el EGFR, proteína transmembranal con un dominio para la unión de ligando extracelular asociada a una protein-tirocinasa intracelular, que regula la proliferación, diferenciación, migración y apoptosis ceIular. Este tratamiento se usa en carcinomas refractarios al tratamiento 0 en pacientes intolerantes a la quimioterapia. Debido al aumento en el interés de nuevas quimioterapias, es importante para el oftalmólogo reconocer y manejar los potenciales efectos secundarios de estos tratamientos ${ }^{12}$.

\section{Características clínicas}

Existe una gran variedad de manifestaciones corneales asociadas a la queratitis tóxica, que van desde una mínima epiteliopatía punteada hasta una ulceración corneal y necrosis ${ }^{13}$. Frecuentemente los pacientes tienen el conocimiento de un agente casual, y refieren historia de un contacto ocular inadvertido (sustancias químicas de limpieza) o a propósito (instilación de un medicamento tópico).

La epiteliopatía tóxica puede ser uni o bilateral y afectar a cualquier individuo, sin importar la edad, la raza o el género. Los pacientes comúnmente pueden presentar algún grado de disconfort ocular, que puede manifestarse como comezón, ardor, fotofobia, sensación de cuerpo extraño o dolor. La visión borrosa y el lagrimeo también se asocian frecuentemente a esta enfermedad ${ }^{14}$.

El hallazgo clínico clásico es la epiteliopatía punteada, que puede o no confluir y muestra un patrón específico en la tinción con fluoresceína, con predominio en el cuadrante inferonasal, donde se produce el tiempo máximo de contacto entre el medicamento y la superficie ocular ${ }^{13}$ (Fig. 2). También se puede observar en algunos casos queratopatía filamentosa 0 pseudodendritas ${ }^{15}$.

El epitelio corneal se puede observar opaco y edematoso, y en ocasiones puede llegar a evidenciarse una queratopatía en huracán ${ }^{16}$. Ocasionalmente puede observarse un patrón de espiral en casos más avanzados (queratopatía verticillata) (Fig. 3). Una exposición severa o prolongada puede llevar a la formación de defectos epiteliales persistentes, queratitis ulcerativa (Fig. 4), adelgazamiento y perforación corneal ${ }^{13,17}$.

\section{Diagnóstico}

Existen varias pruebas que han sido diseñadas para evaluar los efectos tóxicos de las preparaciones oftálmicas sobre la superficie ocular, pero la mayoría no son prácticas, no están disponibles o son difíciles de realizar en el contexto clínico $^{18}$. 


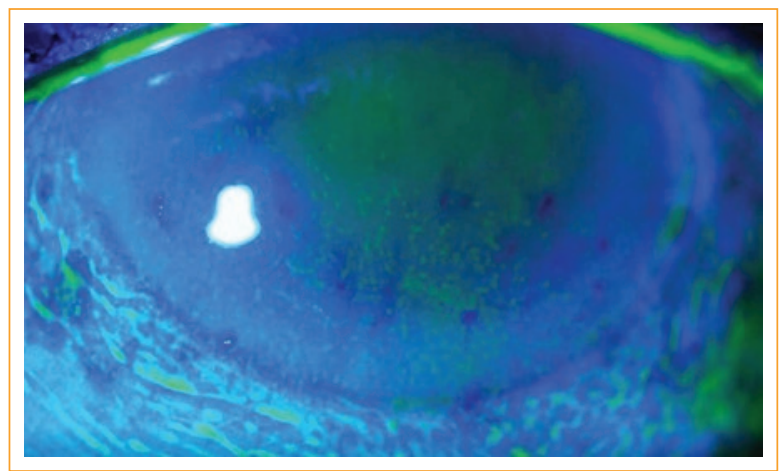

Figura 2. Imagen de una tinción con fluoresceína y azul de cobalto de un paciente con epiteliopatía punteada secundaria a difluprednato; se demuestra la localización inferonasal (imagen tomada del archivo de fotos del Servicio de Córnea y Cirugía Refractiva del Instituto de Oftalmología "Fundación Conde de Valenciana»).

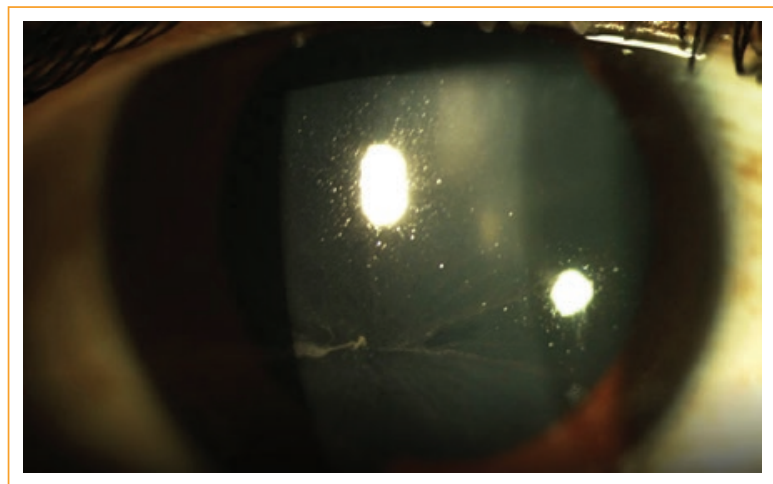

Figura 3. Imagen de la superficie corneal con luz difusa de un paciente con queratopatía verticillata asociada a amiodarona sistémica (tomada del archivo de fotos del Servicio de Córnea y Cirugía Refractiva del Instituto de Oftalmología "Fundación Conde de Valenciana»).

\section{Citología de impresión}

La citología de impresión puede realizarse con anestesia tópica y nos proporciona una capa celular homogénea para estudios histológicos con una arquitectura casi intacta y uniones celulares preservadas. Las células epiteliales, caliciformes e inflamatorias pueden ser diferenciadas con protocolos de inmunotinciones y pueden usarse incluso para análisis citométricos. Se ha demostrado en algunos estudios que los antígenos HLA (Antígenos Leucocitarios Humanos)-DR de clase II y la IL (interleucina)-6, IL-8 e IL-10 son altamente expresados en el epitelio conjuntival de pacientes con historia de tratamiento antiglaucomatoso prolongado ${ }^{19}$.

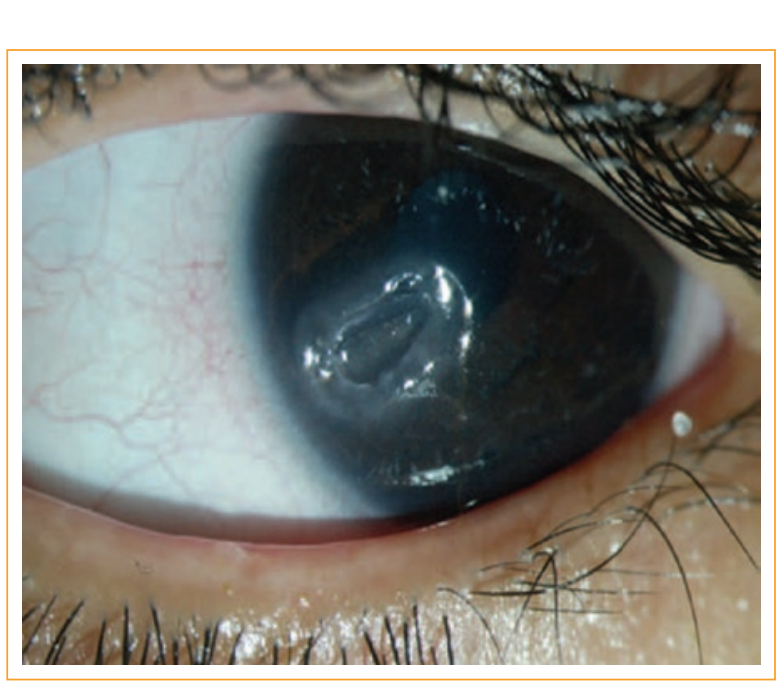

Figura 4. Imagen de una úlcera secundaria al uso prolongado de clorhidrato de tetracaína tópico (tomada del archivo de fotos del Servicio de Córnea y Cirugía Refractiva del Instituto de Oftalmología «Fundación Conde de Valenciana»).

El diagnóstico de epiteliopatía tóxica frecuentemente se basa en la historia clínica y la evolución de la enfermedad, así como en las manifestaciones clínicas actuales. Se debe tomar en cuenta como diagnóstico diferencial cuando los pacientes reportan el inicio de un nuevo tratamiento oftálmico, agente cosmético o producto de lente de contacto durante las semanas previas $^{20}$.

\section{Tratamiento}

Al establecer un agente tóxico, lo más importante en el tratamiento es descontinuar la sustancia o medicamento causal. El tratamiento dependerá del tiempo de exposición, así como de la severidad de la enfermedad de la superficie ocular. Se debe intentar suspender el medicamento cuando es posible o sustituirlo con formulaciones libres de conservadores. Los lentes de contacto deben usarse con precaución, ya que pueden actuar como un reservorio tóxico. En caso de enfermedad moderada, se pueden utilizar lubricantes libres de conservadores para aliviar la sintomatología. En la mayoría de los pacientes sintomáticos puede usarse un esteroide tópico ${ }^{13}$. Una enfermedad más avanzada como un defecto epitelial persistente puede requerir otro tipo de procedimientos como tarsorrafia, injerto de membrana amniótica o colgajos conjuntivales. La queratoplastia puede ser una opción para ulceraciones corneales con inminencia de perforación o necrosis ${ }^{21}$. 


\section{Epiteliopatías nutricionales}

\section{Introducción}

Las enfermedades corneales relacionadas con la nutrición se reconocen desde hace tiempo y son una gran causa de nuevos casos de ceguera cada año.

Las manifestaciones de deficiencia de vitamina $A$ siguen siendo la principal causa de ceguera infantil en los países en desarrollo22. La deficiencia de esta vitamina liposoluble o sus metabolitos se manifiesta de dos maneras: nictalopia y un espectro de enfermedad ocular que se conoce como xeroftalmia, incluyendo queratinización epidérmica y metaplasia escamosa de la córnea y la conjuntiva ${ }^{23}$. La epiteliopatía secundaria a deficiencia de vitamina $A$ es un aspecto clínico muy importante en esta enfermedad.

Por mucho tiempo la vitamina $\mathrm{A}$ ha sido reconocida por mejorar la cicatrización cutánea. Numerosos estudios han demostrado que acelera la migración epitelial y la formación de tejido de granulación, y revierte el retraso en la cicatrización inducida por los corticosteroides ${ }^{24,25}$.

\section{Características clínicas}

La deficiencia de vitamina A promueve una inestabilidad de la capa lagrimal precorneal que provoca una epiteliopatía punteada, que puede progresar a defectos epiteliales, queratinización y edema estromal ${ }^{23}$. Sin tratamiento, los defectos epiteliales progresan a ulceraciones parciales o de espesor completo y pueden desarrollar una infección bacteriana. La queratomalacia es una necrosis licuefactiva del espesor completo de la córnea que, en conjunto con una deficiencia de vitamina $A$, frecuentemente se asocia con un factor sistémico precedente como el sarampión o una desnutrición proteica severa ${ }^{26}$.

Cuando existe actividad de la enfermedad, la córnea puede tener la apariencia de vidrio esmerilado a la retroiluminación (Fig. 5). Esto es más prominente en áreas de queratopatía punteada densa, los mismos sitios en donde el tiempo de ruptura de la película lagrimal es menor. Se pueden observar placas densas irregulares que sugieren una aglomeración de bacterias o queratina fragmentada como en las manchas de Bitot. Las placas, la xerosis y la opacidad corneal se presentan usualmente en ambos ojos (Fig. 6).

\section{Tratamiento}

Los tratamientos recomendados usan ácido transretinoico al $0.01-0.1 \%^{27,28}$. No se ha encontrado mayor

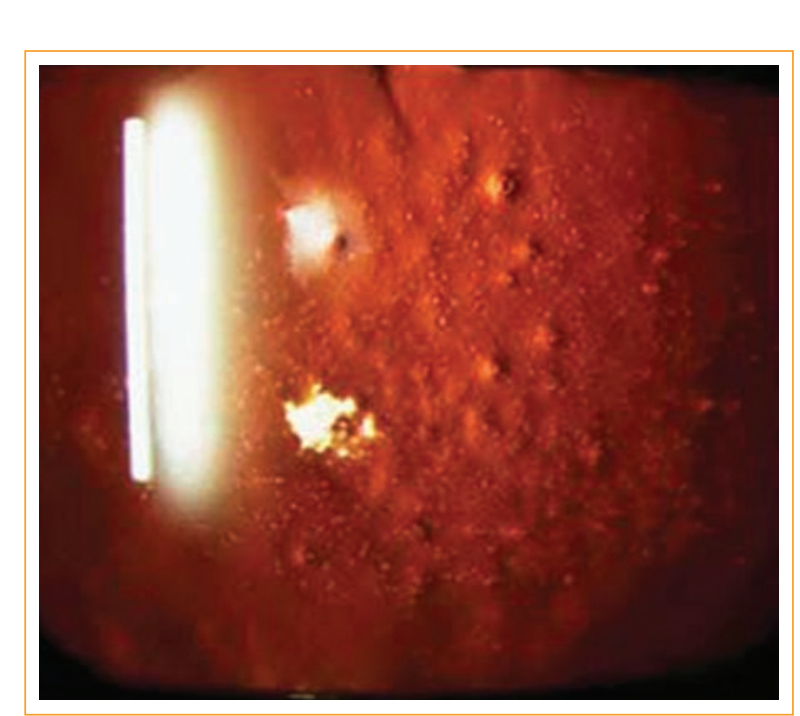

Figura 5. Imagen por retroiluminación de la superficie corneal con apariencia de vidrio esmerilado de una paciente con una epiteliopatía secundaria a una deficiencia de vitamina A (tomada del archivo de fotos del Servicio de Córnea y Cirugía Refractiva del Instituto de Oftalmología "Fundación Conde de Valenciana».

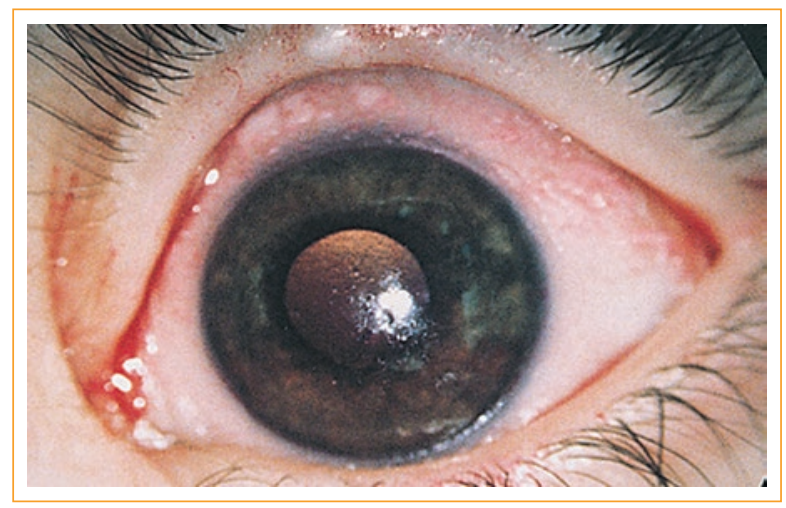

Figura 6. Imagen de la superficie ocular de un paciente con deficiencia de vitamina A con diagnóstico de xeroftalmia (tomada del archivo de fotos del Servicio de Córnea y Cirugía Refractiva del Instituto de Oftalmología "Fundación Conde de Valenciana».

efectividad con dosis más altas $(0.25 \%)^{29}$. Al aumentar la dosis y la frecuencia de aplicación, la probabilidad de desarrollar una disfunción de las glándulas de Meibomio y una blefaroconjuntivitis también aumenta, aunque esto es reversible al descontinuar el ácido retinoico. Los estudios han demostrado que hay que iniciar con tres o cuatro aplicaciones al día, disminuyendo hasta cada dos días conforme los síntomas mejoran (usualmente en 2-3 semanas) ${ }^{30}$. 


\section{Epiteliopatía diabética}

\section{Introducción}

La DM puede conllevar varias complicaciones oculares, entre las cuales se encuentra la epiteliopatía diabética que comprende la queratopatía punteada superficial, erosiones corneales recurrentes y defectos epiteliales persistentes $^{31}$ (Fig. 7). Aunado a esto, la mayoría de los pacientes diabéticos padecen síndrome de ojo seco.

En algunos casos la epiteliopatía diabética es difícil de tratar y puede inducir anormalidades en la cantidad y calidad de la secreción lagrimal, una disminución en la sensibilidad corneal y una pobre adhesión de las células epiteliales regeneradas ${ }^{32}$.

\section{Características clínicas}

Las enfermedades corneales son un cuadro frecuente que engloba varias alteraciones, especialmente epiteliales y endoteliales. La epiteliopatía corneal se manifiesta como queratitis punteada, disminución de la adherencia a la membrana basal e hipoestesia corneal. Tiene interés clínico debido a los síntomas que produce: sensación de cuerpo extraño, ojo rojo, dolor y fluctuación de la visión ${ }^{33}$.

El daño más importante en la córnea se produce en las células de la membrana basal epitelial (con capacidad de regeneración). La córnea diabética sufre asimismo una disfunción nerviosa, lo cual colabora en la epiteliopatía. Las células madre del limbo también están afectadas en la hiperglucemia sostenida, lo que dificulta la regeneración normal del epitelio.

La disminución de la sensibilidad corneal contribuye a la severidad del ojo seco y también predispone a los pacientes a traumas corneales, lo que conlleva un mayor riesgo de desarrollar úlceras corneales neurotróficas y puede afectar adversamente a la cicatrización corneal ${ }^{34}$.

\section{Diagnóstico}

En la literatura algunos investigadores describen que en los pacientes diabéticos las córneas presentan una tendencia a poseer un espesor central mayor estadísticamente significativo ${ }^{35}$, debido a un pleomorfismo y polimegatismo respecto a las córneas de personas que no padecen DM.

\section{Tratamiento}

La epiteliopatía diabética puede tratarse con lubricantes, antibióticos, lentes de contacto terapéuticos y

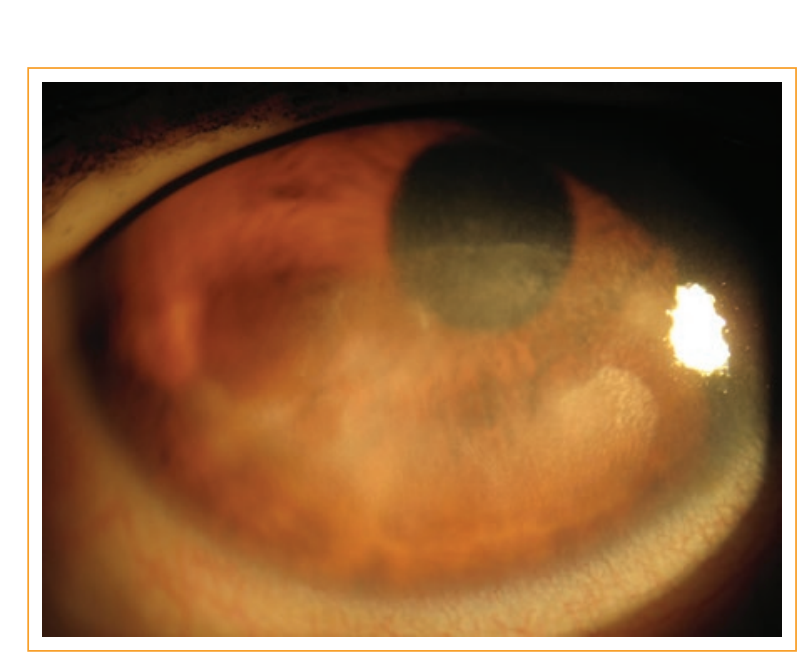

Figura 7. Imagen con luz difusa de la superficie corneal con opacidad subepitelial secundaria a un defecto epitelial persistente en un paciente con DM tipo 2 no controlada (tomada del archivo de fotos del Servicio de Córnea y Cirugía Refractiva del Instituto de Oftalmología "Fundación Conde de Valenciana».

tarsorrafia, todos los tratamientos que generen condiciones más favorables para la cicatrización. Las fallas de los métodos convencionales para mejorar la reparación epitelial no sólo están relacionadas con mayores síntomas de dolor y malestar para el paciente, sino que proveen una ventana para infecciones que pueden conllevar problemas devastadores para la visión. Además, ninguna de estas terapias está dirigida a la patofisiología del retraso en la reepitelización corneal secundaria a DM, por lo que es de vital importancia el control metabólico del paciente ${ }^{36}$.

Algunos estudios han demostrado que los factores de crecimiento y las citosinas pueden mejorar significativamente la epitelización (proliferación epitelial y migración) y, consecuentemente, acelerar la cicatrización epitelial. Más recientemente se ha demostrado la mejoría y el aumento significativo de la tasa de cicatrización corneal con la administración tópica de insulina, naltrexona y nicergolina ${ }^{37}$.

\section{Conclusión}

Existen diversas causas de epiteliopatía corneal, por lo que es importante conocer las más frecuentes para su adecuado diagnóstico y tratamiento. Dentro de las causas poco frecuentes de epiteliopatía corneal encontramos las tóxicas, nutricionales e inflamatorias, y la diabética es la más importante de estas últimas. Estas condiciones se deben buscar intencionadamente en la 
práctica oftalmológica en los casos de epiteliopatías persistentes o refractarias al tratamiento convencional. Es obligación del oftalmólogo conocer e identificar estas causas para su adecuado manejo y para evitar así complicaciones que pueden afectar a la visión de manera irreversible.

\section{Bibliografía}

1. Edelhauser HF, Ubels J. The cornea and the sclera. En: Kaufman PL, Alm A, eds. Adler's Physiology of the Eye. 10. ${ }^{a}$ ed. St. Louis, MO: Mosby; 2003. p. 47-114.

2. Grant WM. Toxicology of the Eye. Springfield, IL: Charles C Thomas; 1986.

3. Chen W, Li Z, Hu J, Zhang Z, Chen L, Chen Y, Liu Z. Corneal alternations induced by topical application of benzalkonium chloride in rabbit. PLoS One. 2011;6(10):e26103.

4. Chen HT, Chen KH, Hsu WM. Hsu Toxic keratopathy associated with abuse of low-dose anesthetic: a case report. Cornea. 2004;23(5):527-9.

5. Burstein NL. Corneal toxicity of topically applied drugs, vehicles and preservatives. Surv Ophthalmol. 1980;25(1):15-30.

6. De Saint Jean M, Brignole F, Bringuier AF, Bauchet A, Feldmann G, Baudouin $C$. Effects of benzalkonium chloride on grows and survival of Chang conjunctival cells. Invest Ophthalmol Vis Sci. 1999;40(3):619-30.

7. Vignesh AP, Srinivasan R, Karanth S. A case report of severe corneal toxicity following $0.5 \%$ topical moxifloxacin use Case Rep. Ophthalmol. 2015;6(1):63-5

8. Mian SI, Gupta A, Pineda R $2^{\text {nd }}$. Corneal ulceration and perforation with ketorolac tromethamine (Aculr R) use after PRK. Cornea. 2006:25(2):232-4

9. Kim YJ, Payal AR, Daly MK. Effects of tear gases on the eye. Surv Ophthalmol. 2016;61(4):434-42.

10. Spector J, Fernandez WG. Chemical, thermal, and biological ocular exposures. Emerg Med Clin North Am. 2008;26(1):125-36, vii.

11. Goldman DR, Seefeld AW. Ocular toxicity associated with indirect exposure to African spitting cobra venom. Wilderness Environ Med. 2010;21(2): 134-6.

12. Saint-Jean A, Sainz de la Maza M, Morral M, Torras J, Quintana R, Molina JJ, et al. Ocular adverse events of systemic inhibitors of the epidermal growth factor receptor: report of 5 cases. Ophthalmology. 2012:119(9):1798-802.

13. Graue-Hernández EO, Navas A, Ramírez-Miranda A. Toxic keratoconjunctivitis. En: Holland EJ, Mannis MJ, Lee WB, eds. Ocular surface disease: cornea, conjunctiva and tear film. Atlanta: Elsevier; 2013. p. 189-93.

14. Dart J. Corneal toxicity: the epithelium and stroma in iatrogenic and factitious disease. Eye (Lond). 2003;17(8):886-92.

15. Patel S, Dhakhwa K, Rai SKC, Bhattarai B, A Pandey, BP Bandhu. Clinical profile of toxic keratoconjunctivitis after ocular trauma with insect. Nep JOL. 2013;1(4):41-4.

16. Wilson FM $2^{\text {nd }}$. Adverse external ocular effects of topical ophthalmic therapy: an epidemiologic, laboratory, and clinical study. Trans Am Ophthalmol Soc 1983;81:854-965.
17. Tok OY, Tok L, Atay IM, Argun TC, Demirci N, Gunes A. Toxic keratopathy associated with abuse of topical anesthetics and amniotic membrane transplantation for treatment. Int J Ophthalmol. 2015;8(5):938-44.

18. Barbaro V, Ferrari S, Fasolo A, Pedrotti E, Marchini G, Sbabo A, et al. Evaluation of ocular surface disorders: a new diagnostic tool based on impression cytology and confocal laser scanning microscopy. $\mathrm{Br} \mathrm{J}$ Ophthalmol. 2010;94(7):926-32.

19. Reilly CD, Mannis MJ. Toxic conjunctivitis. En: Krachmer JH, Mannis MJ, Holland EJ, eds. Cornea - Fundamentals, Diagnosis and Management. St. Louis: Mosby; 2011. p. 613-21.

20. Altinok AA, Balikoglu M, Sen E, Serdar K. Nonpreserved amniotic membrane transplantation for bilateral toxic keratopathy caused by topical anesthetic abuse: a case report. J Med Case Reports. 2010;4:262.

21. Steinkuller PG, Du L, Gilbert C, Foster A, Collins ML, Coats DK. Childhood blindness. J AAPOS. 1999;3(1):26-32.

22. Smith J, Steinemann TL. Vitamin A deficiency and the eye. Int Ophthalmol Clin. 2000;40(4):83-91.

23. Ehrlich HP, Tarver H, Hunt TK. Effects of vitamin A and glucocorticoids upon inflammation and collagen synthesis. Ann Surg. 1973;177(2):222-7.

24. Herbort CP, Weissman SS, Ostler HB, Cevallos A, Char DH. Ocular surface keratinization as a predictor of response to topical retinoic acid therapy. Arch Ophthalmol. 1989;107:1275-6.

25. Sommer A. Effects of vitamin A deficiency on the ocular surface. Ophthalmology. 1983;90(6):592-600.

26. Chander A, Chopra R, Batra N. Vitamin A deficiency: an eye sore. J Med Nutr Nutraceut. 2013;2:41.

27. Asselineau D, Bernard BA, Bailly C, Darmon M. Retinoic acidimproves epidermal morphogenesis. Dev Biol. 1989;133(2):322-35.

28. Herbort CP, Zografos L, Zwingli M, Schoeneich $M$. Topical retinoic acid in dysplastic and metaplastic keratinization of corneoconjunctival epithelium. Graefes Arch Clin Exp Ophthalmol. 1988;226(1):22-6.

29. Kruse FE, Tseng SC. Retinoic acid regulates clonal growth and differentiation of cultured limbal and peripheral corneal epithelium. Invest Ophthalmol Vis Sci. 1994;35(5):2405-20.

30. Zouboulis CC. Isotretinoin revisited: pluripotent effects on human sebaceous gland cells. J Invest Dermatol. 2006;126(10):2154-6.

31. Saini JS, Khandalavla B. Corneal epithelial fragility in diabetes mellitus. Can J Ophthalmol. 1995;30(3):142-6.

32. Inoue K, Kato S, Ohara C, Numaga J, Amano S, Oshika T. Ocular and systemic factors relevant to diabetic keratoepitheliopathy. Cornea. 2001;20(8):798-801.

33. McNamara NA, Brand RJ, Polse KA, Bourne WM. Corneal function during normal and high serum glucose levels in diabetes. Invest Ophthalmol Vis Sci. 1998;39(1):3-17

34. Hyndiuk RA, Kazarian EL, Schultz Rom Seideman S. Neurotrophic corneal ulcers in diabetes mellitus. Arch. Ophthalmol. 1977;95(12):2193-6.

35. Inoue $\mathrm{K}$, Kato S, Inoue $\mathrm{Y}$, Amano S, Oshika T. The corneal endothelium and thickness in type II diabetes mellitus. Jpn J Ophthalmol. 2002;46(1): 65-9.

36. Kaji Y. Prevention of diabetic keratopathy. Br J Ophthalmol. 2005;89(3): 254-5.

37. Abdelkader H, Patel DV, McGhee CNj, Alany RG. New therapeutic approaches in the treatment of diabetic keratopathy: a review. Clin Exp Ophthalmol. 2011;39(3):259-70. 